\title{
DESIGN OF TARGETING COST FUNCTION FOR EVOLUTIONARY OPTIMIZATION OF CHAOS CONTROL
}

\author{
Roman Senkerik \\ Ivan Zelinka \\ Eduard Navratil \\ Department of Applied Informatics \\ Faculty of Applied Informatics, Tomas Bata University in Zlin \\ Nad Stranemi 4511, Zlin 762 72, Czech Republic \\ E-mail: \{senkerik, zelinka, enavratil\}@fai.utb.cz
}

\section{KEYWORDS}

Chaos, control, optimization, evolutionary algorithms, parameter estimation

\begin{abstract}
This contribution deals with optimization of the control of chaos by means of evolutionary algorithms. The main aim of this work is to show that evolutionary algorithms are capable of optimization of chaos control and to show several methods of constructing the complex targeting cost function leading to satisfactory results. As a model of deterministic chaotic system the two dimensional Henon map was used. The optimizations were realized in several ways, each one for another cost function or another desired periodic orbit. The evolutionary algorithm Self-Organizing Migrating Algorithm (SOMA) was used in four versions. For each version, simulations were repeated several times to show and check robustness of used method and cost function. At the end of this work the results of optimized chaos control for each designed targeting cost function are compared.
\end{abstract}

\section{INTRODUCTION}

These days evolutionary algorithms (EA) are known as powerful tools for almost any difficult and complex optimization problem. But the quality of optimization process results mostly depends on proper design of used cost function, especially when the EAs are used for optimization of chaos control. It is well known that deterministic chaos in general and also any technique to control of chaos are sensitive to parameter setting, initial conditions and in the case of optimization they are also extremely sensitive to the construction of used cost function.

In this work the Pyragas's delayed feedback control technique (Just 1999, Pyragas 1992) was used. Unlike the original OGY control method (Ott, et al. 1990) it can be simply considered as targeting and stabilizing algorithm together in one package (Kwon 1999). Another big advantage of Pyragas method is the amount of accessible control parameters. This is very advantageous for successful use of optimization of parameters setting by means of EA, leading to improvement of system behavior and better and faster stabilization to the desired periodic orbits. Some research in this field has been recently done using the evolutionary algorithms for optimization of local control of chaos (Richter and Reinschke, 2000).

This contribution deals with an investigation on the design of the targeting cost function securing the improvement of system behavior and faster stabilization to desired periodic orbits. The control law is based on Pyragas method: Extended delay feedback control ETDAS (Pyragas 1995). This contribution is continuation of previous experiments with application of EA to chaos control (Zelinka 2005a, Zelinka 2005b, Senkerik 2006a, Senkerik 2006b).

\section{PROBLEM DESIGN}

\section{Problem selection and case studies}

The chosen example of chaotic system was two dimensional Henon map in form (1).

$$
\begin{aligned}
& x_{n+1}=a-x_{n}^{2}+b y_{n} \\
& y_{n+1}=x_{n}
\end{aligned}
$$

This work primarily consists of two case studies. Both of them are focused on estimation of three accessible control parameters for EDTAS method to stabilize desired UPO (unstable periodic orbit) and comparison of results for used cost function. Desired UPOs are following: p-1 (a fixed point) in the first case and p-2 in the second case. All simulations were 50 times repeated for each EA version. The control method - ETDAS in the discrete form suitable for two dimensional Henon map has form (2).

$$
\begin{gathered}
x_{n+1}=a-x_{n}^{2}+b y_{n}+F_{n} \\
F_{n}=K\left[(1-R) S_{n-m}-x_{n}\right] \\
S_{n}=x_{n}+R S_{n-m}
\end{gathered}
$$

Where $K$ and $R$ are adjustable constants, $F$ is the perturbation, $S$ is given by a delay equation utilizing previous states of the system and $m$ is the period of $m$ - 
periodic orbit to be stabilized. The perturbation $F_{n}$ in equations (2) may have arbitrarily large value, which can cause diverging of the system outside the interval $\{-$ $1.5,1.5\}$. Therefore, $F_{n}$ should have a value between $-F_{\max }, F_{\max }$ and EA should find an appropriate value of this limitation to avoid diverging of system.

\section{The cost function}

In this work several types of cost function (CF) were developed and tested for stabilization of $\mathrm{p}-1$ orbit (fixed point) and $\mathrm{p}-2$ orbit. The $\mathrm{CF}$ has been calculated in general from the distance between desired state and actual system output. The minimal value of this cost function revealing the best solution is zero. The aim of all the simulations was to find the best solution that returns the cost function value as close as possible to zero. The idea was to minimize the area created by the difference between the required state (stabilized fixed point) and the real system output on the whole simulation interval $-\tau$, thus this proposal of CF should secure fast targeting into the close neighborhood of $\mathrm{p}-1$ orbit and its stabilization. The CF1 is given by (3).

$C F 1=\sum_{t=0}^{\tau}\left|T S_{t}-A S_{t}\right|$

Where: TS - target state, AS - actual state

Another cost function (CF2) had to be used for stabilizing of higher periodic orbit. It was synthesized from the simple CF1 (3) and other terms were added. In this case it is not possible to use the simple rule of minimizing the area created by the difference between the required and actual state on the whole simulation interval $-\tau$, due to the many serious reasons, for example: degrading of the possible best solution by phase shift of periodic orbit. This CF is in general based on searching for desired stabilized periodic orbit and thereafter calculation of the difference between desired and found actual periodic orbit on the short time interval - $\tau$ s (approx. 20 - 50 iterations) from the point, where the first min. value of difference between desired and actual system output is found. Such a design of CF should secure the successful stabilization of higher periodic orbit anywise phase shifted. This CF can also be used for p-1 orbit. The CF2 has form (4).

$C F 2=$ penalization $1+\sum_{t=\tau 1}^{\tau 2}\left|T S_{t}-A S_{t}\right|$

Where: $\tau 1$ - the first min. value of difference between

TS and AS

$\tau 2$ - the end of optimizing interval $(\tau 1+\tau \mathrm{s})$

penalization $1=0$ if $\tau-\tau 2 \geq \tau \mathrm{s}$;

penalization $1=10^{*}(\tau-\tau 2)$ if $\tau-\tau 2<\tau$ s (i.e. late stabilization)

\section{Design of targeting $\mathrm{CF}$}

To decrease the average iterations needed for stabilization (IStab value) it was necessary to modify the definition of CF. The CF1 is not suitable for adding any term of penalization for slowly stabilizing solutions, thus the CF2 was modified to use for both p-1 and p-2 orbit. The CF value is multiplied by number of iterations $(N I)$ of the first found minimal value of difference between desired and actual system output (i.e. the beginning of fully stabilized UPO). To avoid any problem with $\mathrm{CF}$ returning value 0 and to put the penalization to similar level as the not-penalized $\mathrm{CF}$ value, the small constant $(S C)$ is added to $\mathrm{CF}$ value before penalization (multiplying by $N I$ ). The modified CF2 has form (5).

$$
C F_{\text {TARG }}=N I\left(S C+\text { penalization } 1+\sum_{t=\tau 1}^{\tau 2}\left|T S_{t}-A S_{t}\right|\right)
$$

Where:

$$
S C=10^{-16} \text { for } \mathrm{p}-1 \text { orbit; } S C=10^{-8} \text { for } \mathrm{p}-2 \text { orbit }
$$

This proposal of CF was successfully tested in previous experiments. To avoid any problems with defining the value of $S C$ in advance (especially for stabilization of higher periodic orbit) the design of new targeting $\mathrm{CF}$ had to be changed. The first version of final design of targeting $\mathrm{CF}$ ( $\left.\mathrm{CF}_{\mathrm{TARG} 1}\right)$ has form (6). The $S C$ value (7) is here counted with the aid of power of not-penalized basic part of CF.

$$
C F_{\text {TARG1 }}=N I\left(S C+\text { penalization } 1+\sum_{t=\tau 1}^{\tau 2}\left|T S_{t}-A S_{t}\right|\right)
$$

Where:

$$
\begin{aligned}
& S C=10^{\mathrm{EXPCF}} \\
& E X P C F=\log _{10}\left(\sum_{t=\tau 1}^{\tau 2}\left|T S_{t}-A S_{t}\right|+10^{-15}\right)
\end{aligned}
$$

In the second version of final design $\left(\mathrm{CF}_{\mathrm{TARG} 2}\right)$, there is only slight change in comparison with previous design. Here the number of steps for stabilization (NI) multiplies only the small constant $(S C)$ which is counted in the same way as in the previous case (7) The second version of final design of targeting $\mathrm{CF}\left(\mathrm{CF}_{\mathrm{TARG} 2}\right)$ has form (8)

$$
C F_{\text {TARG } 2}=(N I \cdot S C)+\text { penalization } 1+\sum_{t=\tau 1}^{\tau 2}\left|T S_{t}-A S_{t}\right|
$$

These two targeting CFs (6) and (8) are tested and compared in this work. The difference between them can be clearly seen on Fig. 1, which shows the dependence of $\mathrm{CF}_{\mathrm{TARG} 1}$ (6) and $\mathrm{CF}_{\mathrm{TARG} 2}$ (8) values on the adjustable parameter $K$ and $F_{\text {max }}$. 

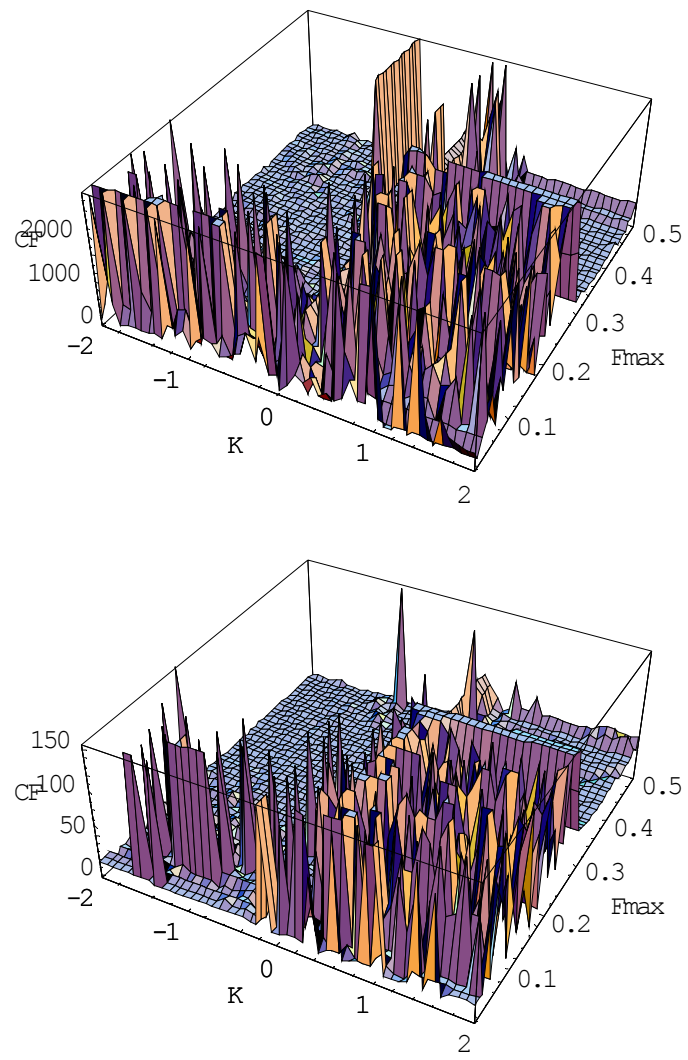

Fig. 1. Dependence of CF value on parameters $K$ and $F_{\text {max }}$ for p-2 orbit, $x_{\text {initial }}=0.7, \mathrm{CF}_{\mathrm{TARG} 1}$ (upper, $R=0.2928), \mathrm{CF}_{\mathrm{TARG} 2}($ lower, $R=0.4636$ )

\section{Optimization algorithms}

For the experiments described here, stochastic optimization algorithm SOMA (Zelinka, 2004), has been used. It was chosen because it has been proven that this algorithm has the ability to converge towards the global optimum.

\section{EXPERIMENTAL RESULTS}

Four versions of SOMA were used for all simulations. See Table 1 for relation between each version and index mark in Figures. Parameters for optimizing algorithm were set up the way in order to reach the same value of maximal $\mathrm{CF}$ evaluations for all used versions. Each version of SOMA has been applied 50 times in order to find the actual optimum. The primary aim of this contribution is not to show which version is better or worse but to show that the EA can in reality be used for deterministic chaos control when the cost function is properly defined and how the results can be influenced by even slight changes in the design of $\mathrm{CF}$. All results are shown only for variable $x$ of two dimensional Henon map because of its form (1), where the variable $y$ has the same values as variable $x$ but it is only phase shifted.
The ranges of all estimated parameters were these: $-2 \leq K \leq 2,0 \leq F_{\max } \leq 0.5$ and $0 \leq R \leq 1$

Table 1: Used versions of SOMA

\begin{tabular}{|c|c|}
\hline Index & Algorithm / Version \\
\hline 1 & SOMA AllToOne \\
\hline 2 & SOMA AllToRandom \\
\hline 3 & SOMA AllToAll \\
\hline 4 & SOMA AllToAllAdaptive \\
\hline
\end{tabular}

The best solution for each version of SOMA are shown in Tables together with other optimization results like Iterations required for stabilization (IStab) Average IStab value for 50 repeated simulations (Avg. IStab). Comparison of SOMA versions from the point of IStab, and estimated parameters $K, F_{\max }, R$ is in Figures. These diagrams show the variance of observed parameter and the small rectangular mark represents average value.

\section{Control of chaos, p-1 orbit, $\mathrm{CF}_{\text {TARG1 }}$}

This case is focused on the stabilization of p-1 orbit. Unperturbed Henon map has this p-1 orbit: $x_{\mathrm{F}}=0.8$

Each SOMA version gave almost the same result of CF value for the best solution. See Fig. 2 for the best individual solution with the lowest CF value (SOMA ATO) and Fig. 3 for comparison of all used versions. Based on obtained results, it may be stated that the control parameters estimated in the optimizations ensured fast reaching of a desired state, on average, about 50 iterations are required.

Table 2: Results for $\mathrm{p}-1$ orbit, $\mathrm{CF}_{\mathrm{TARG} 1}$

\begin{tabular}{|c|c|c|c|c|}
\hline EA & 1 & 2 & 3 & 4 \\
\hline$K$ & -0.8543 & -0.8367 & -0.8564 & -0.8543 \\
\hline$F_{\max }$ & 0.1863 & 0.4293 & 0.3899 & 0.1863 \\
\hline$R$ & 0.2098 & 0.2010 & 0.2099 & 0.20978 \\
\hline CF Val. & $3.9 \cdot 10^{-15}$ & $4.0 \cdot 10^{-15}$ & $4.2 \cdot 10^{-15}$ & $3.9 \cdot 10^{-15}$ \\
\hline $\begin{array}{c}\text { IStab } \\
\text { Best sol. }\end{array}$ & 39 & 40 & 42 & 39 \\
\hline $\begin{array}{c}\text { Avg. } \\
\text { IStab }\end{array}$ & $\mathbf{5 0}$ & $\mathbf{5 1}$ & $\mathbf{4 9}$ & $\mathbf{4 9}$ \\
\hline
\end{tabular}

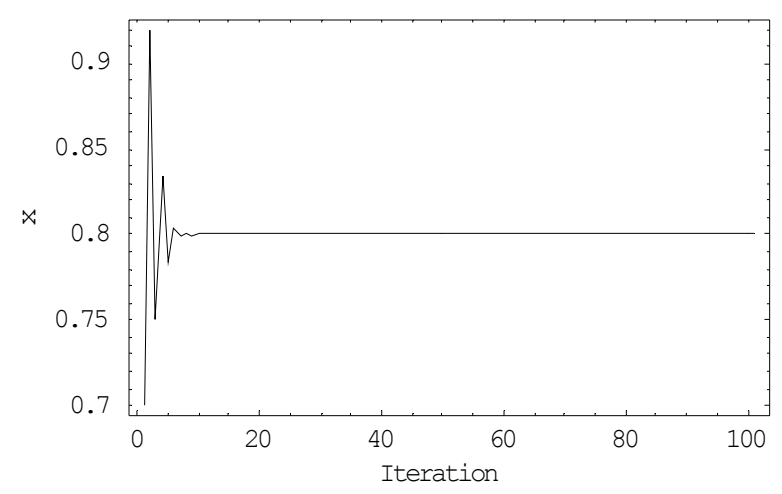

Fig. 2. Best individual solution: $\mathrm{p}-1$ orbit, $\mathrm{CF}_{\mathrm{TARG} 1}$ 

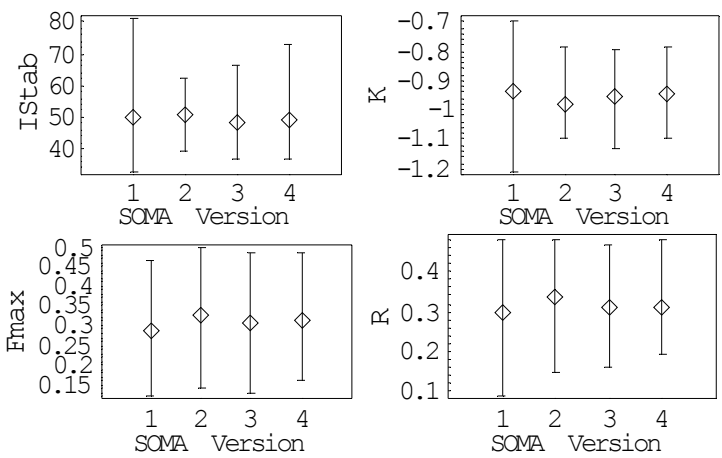

Fig. 3. Comparison of SOMA versions, $\mathrm{p}-1, \mathrm{CF}_{\mathrm{TARG} 1}$

\section{Control of chaos, p-1 orbit, $\mathbf{C F}_{\text {TARG2 }}$}

The best results of each SOMA version are shown in Table 3. The best solution and comparison of SOMA versions are depicted in Fig. 4 and Fig. 5. As can be seen from Table 3, three SOMA versions give similar results of the best solution; SOMA ATA has found the lowest $\mathrm{CF}$ value. From the comparison with previous case (See Table 2) follows, that the average IStab value is smaller. But on the other hand this CF give two best solutions (SOMA ATO and ATAA), where the final CF value is not divisible by the $N I$ value (or IStab) without remainder Thus this design of $\mathrm{CF}$ secures very fast reaching of desired state but with slightly lower quality of stabilization (basic part of $\mathrm{CF}>0$ ) For stabilization, on average, about 38 iterations are needed

Table 3: Results for $\mathrm{p}-1$ orbit, $\mathrm{CF}_{\mathrm{TARG} 2}$

\begin{tabular}{|c|c|c|c|c|}
\hline EA & 1 & 2 & 3 & 4 \\
\hline$K$ & -0.7525 & -0.8640 & -0.8498 & -0.7619 \\
\hline$F_{\max }$ & 0.4155 & 0.2692 & 0.1462 & 0.1861 \\
\hline$R$ & 0.1316 & 0.2313 & 0.2055 & 0.1343 \\
\hline CF Val. & $4.07 \cdot 10^{-15}$ & $4.2 \cdot 10^{-15}$ & $3.8 \cdot 10^{-15}$ & $3.97 \cdot 10^{-15}$ \\
\hline $\begin{array}{c}\text { IStab } \\
\text { Best sol. }\end{array}$ & 34 & 42 & 38 & 33 \\
\hline $\begin{array}{c}\text { Avg. } \\
\text { IStab }\end{array}$ & $\mathbf{3 8}$ & $\mathbf{3 9}$ & $\mathbf{3 9}$ & $\mathbf{3 8}$ \\
\hline
\end{tabular}

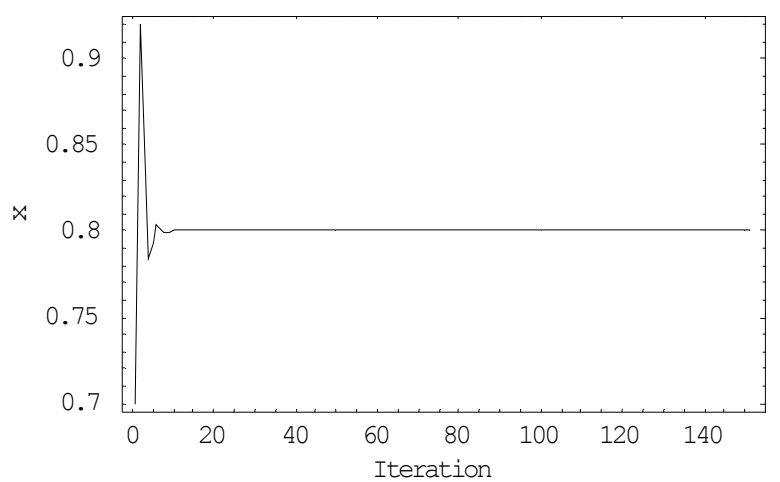

Fig. 4. Best individual solution: $\mathrm{p}-1$ orbit, $\mathrm{CF}_{\mathrm{TARG}}$
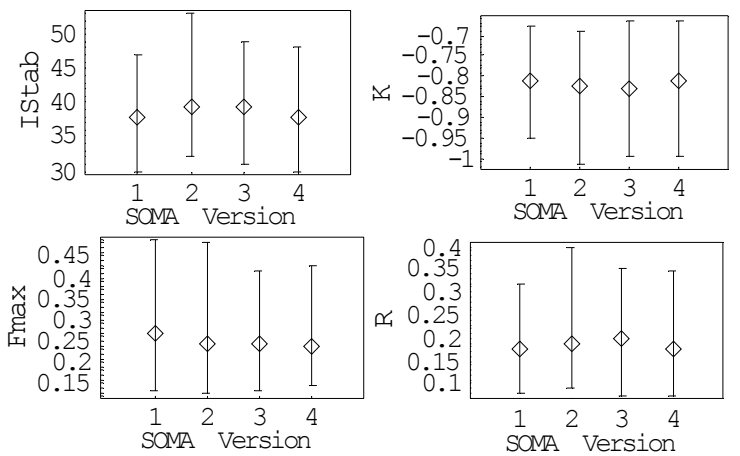

Fig. 5. Comparison of SOMA versions, $\mathrm{p}-1, \mathrm{CF}_{\mathrm{TARG} 2}$

\section{Control of chaos, p-2 orbit, $\mathrm{CF}_{\text {TARG1 }}$}

This case is focused on the stabilization of $2 p$-orbit. Unperturbed Henon map has this p-2 orbit: $x_{1}=-0.56$, $x_{2}=1.26$.

In comparison with $\mathrm{p}$-1 orbit the EDTAS control method is not able to reach "exact" stabilization of p-2 orbit. Thus the not penalized and multiplied basic CF value is always greater than zero. From Table 4 it follows that all versions of SOMA have found relatively similar results for the best solution, from the point of view of $\mathrm{CF}$ value. See Fig. 6 for the best individual solution with the lowest CF value (SOMA ATR) and Fig. 7 for comparison of SOMA versions. For successful stabilization of p- 2 orbit, on average, about 84 iterations are needed

Table 4: Results for $\mathrm{p}-2$ orbit, $\mathrm{CF}_{\mathrm{TARG} 1}$

\begin{tabular}{|c|c|c|c|c|}
\hline EA & 1 & 2 & 3 & 4 \\
\hline$K$ & 0.4074 & 0.4056 & 0.3646 & 0.3873 \\
\hline$F_{\max }$ & 0.1990 & 0.1762 & 0.1474 & 0.1764 \\
\hline$R$ & 0.2768 & 0.2928 & 0.2079 & 0.2666 \\
\hline CF Val. & $2.57 \cdot 10^{-5}$ & $3.12 \cdot 10^{-6}$ & $9.01 \cdot 10^{-6}$ & $1.46 \cdot 10^{-5}$ \\
\hline $\begin{array}{c}\text { IStab } \\
\text { Best sol. }\end{array}$ & 123 & 129 & 130 & 127 \\
\hline $\begin{array}{c}\text { Avg. } \\
\text { IStab }\end{array}$ & $\mathbf{9 2}$ & $\mathbf{8 0}$ & $\mathbf{8 3}$ & $\mathbf{8 0}$ \\
\hline
\end{tabular}

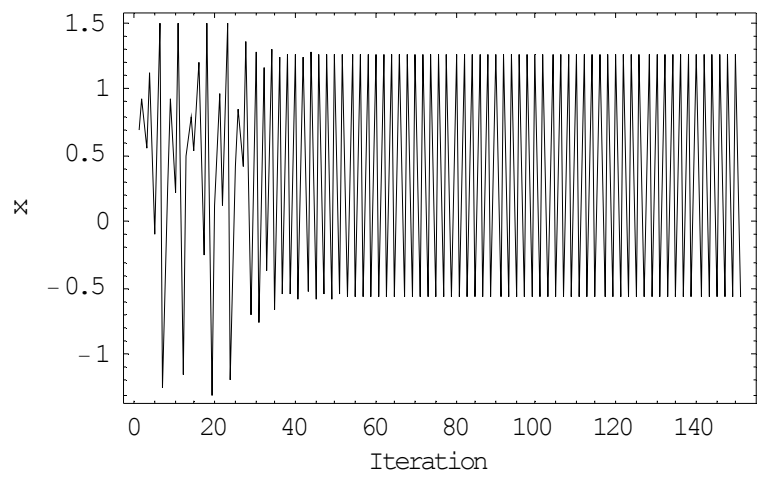

Fig. 6. Best individual solution: $\mathrm{p}-2$ orbit, $\mathrm{CF}_{\mathrm{TARG} 1}$ 

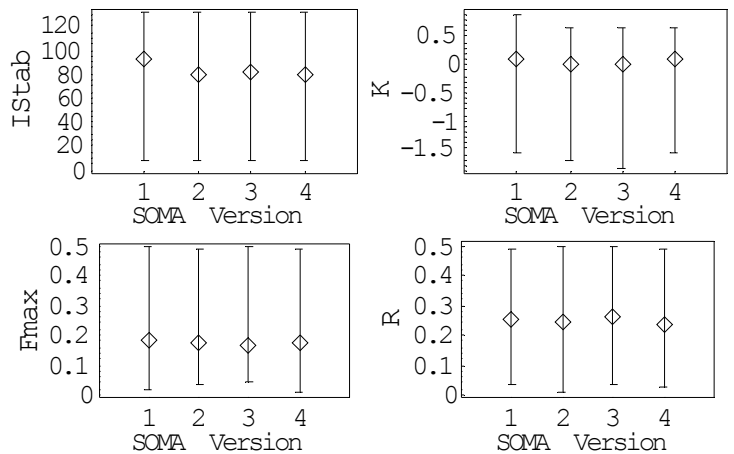

Fig. 7. Comparison of SOMA versions, $\mathrm{p}-2, \mathrm{CF}_{\mathrm{TARG} 1}$

\section{Control of chaos, p-2 orbit, $\mathrm{CF}_{\mathrm{TARG} 2}$}

See Table 5 for the results of this optimization. The best individual solution with the lowest $\mathrm{CF}$ value (SOMA ATR) is depicted on Fig. 8. and comparison of SOMA versions on Fig. 9. In spite of the promising results in case of p-1 orbit, smaller final $\mathrm{CF}$ values for the best solutions and less nonlinear CF surface (see Fig. 1) it seems that this optimization by means of $\mathrm{CF}_{\mathrm{TARG} 2}$ gives worse results than previous optimization from the point of view of average IStab value. For successful stabilization of p-2 orbit in this case, on average, about 93 iterations are needed

Table 5: Results for $\mathrm{p}-2$ orbit, $\mathrm{CF}_{\mathrm{TARG} 2}$

\begin{tabular}{|c|c|c|c|c|}
\hline EA & 1 & 2 & 3 & 4 \\
\hline$K$ & 0.4981 & 0.3535 & 0.5660 & 0.3965 \\
\hline$F_{\max }$ & 0.1414 & 0.1350 & 0.1689 & 0.1799 \\
\hline$R$ & 0.3867 & 0.1728 & 0.4636 & 0.2919 \\
\hline CF Val. & $1.53 \cdot 10^{-5}$ & $2.21 \cdot 10^{-6}$ & $1.47 \cdot 10^{-8}$ & $1.53 \cdot 10^{-7}$ \\
\hline $\begin{array}{c}\text { IStab } \\
\text { Best sol. }\end{array}$ & 131 & 124 & 130 & 127 \\
\hline $\begin{array}{c}\text { Avg. } \\
\text { IStab }\end{array}$ & $\mathbf{8 5}$ & $\mathbf{8 9}$ & $\mathbf{1 0 1}$ & $\mathbf{9 7}$ \\
\hline
\end{tabular}

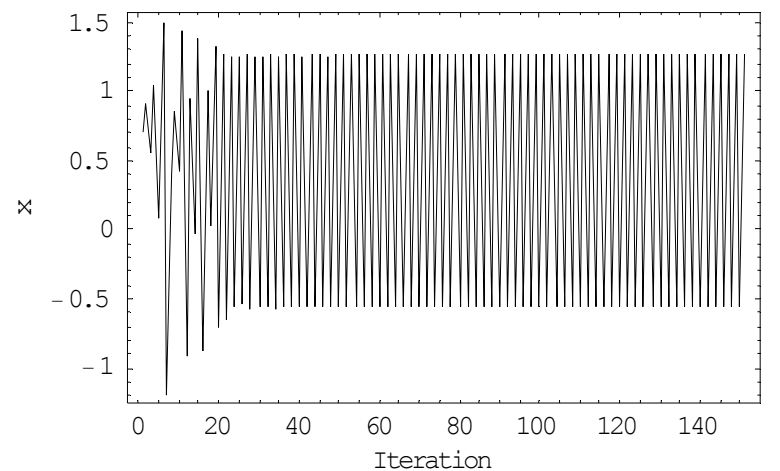

Fig. 8. Best individual solution: $\mathrm{p}-2$ orbit, $\mathrm{CF}_{\mathrm{TARG} 2}$
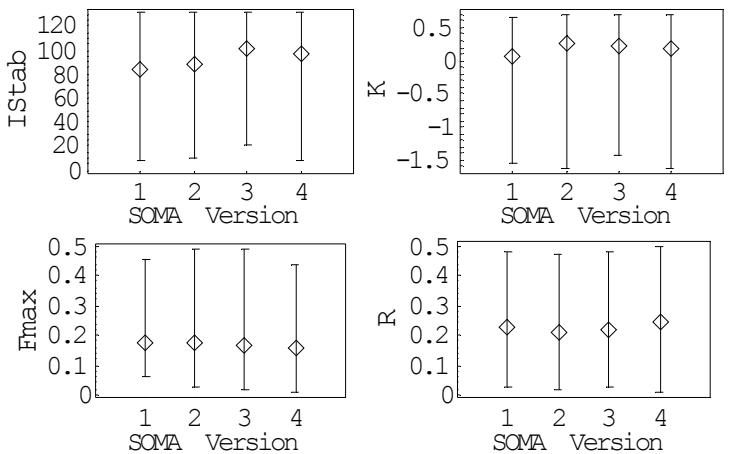

Fig. 9. Comparison of SOMA versions, $\mathrm{p}-2, \mathrm{CF}_{\mathrm{TARG} 2}$

\section{COMPARISON OF RESULTS}

The comparison of results for $\mathrm{CF}_{\mathrm{TARG} 1}(6)$, and $\mathrm{CF}_{\mathrm{TARG} 2}$ (8) with initial conditions uniformly distributed in the region of $0<x_{\text {initial }}<1$ was done for these two cases: p-1 orbit and p-2 orbit. In the first case (Fig. 10) the control algorithm was set up as follows: For $\mathrm{CF}_{\mathrm{TARG} 1}$ as the solution given by SOMA ATR (Table 2.) and for $\mathrm{CF}_{\mathrm{TARG} 2}$ as the solution given by SOMA ATO (Table 3.).

In the second case (Fig. 11) the control algorithm was set up as follows: For $\mathrm{CF}_{\mathrm{TARG} 1}$ as the best solution given by SOMA ATR (Table 4.) and for $\mathrm{CF}_{\mathrm{TARG} 2}$ as the best solution given by SOMA ATR (Table 5.).

As can be seen from Figures 10 and 11 both targeting CFs steers the system very quickly to desired state. In case of $\mathrm{p}-1$ orbit, the results are very similar, but paradoxically in case of $\mathrm{p}-2$ orbit the solution obtained by $\mathrm{CF}_{\mathrm{TARG} 2}$ gives better results for this comparison, although this CF gives the worse results of Avg.Istab value for 50 repeated optimizations (See Table 4 and 5).
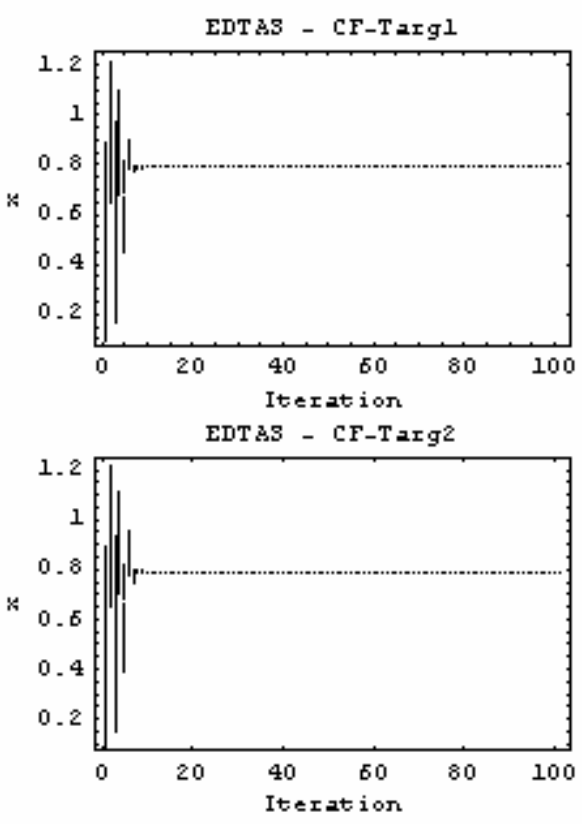

Fig. 10. Comparison of the best solutions, p-1orbit, $\mathrm{CF}_{\text {TARG1 }}$ (upper) and $\mathrm{CF}_{\mathrm{TARG} 2}$ (lower) 

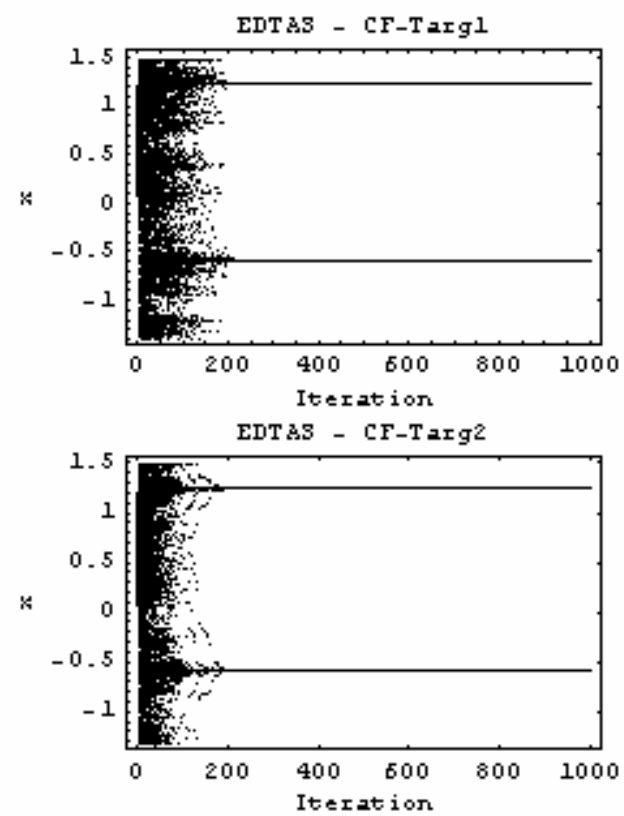

Fig. 11. Comparison of the best solutions, $\mathrm{p}-2$ orbit, $\mathrm{CF}_{\text {TARG1 }}$ (upper) and $\mathrm{CF}_{\text {TARG2 }}$ (lower)

\section{CONCLUSION}

Based on obtained results, it may be claimed that all simulations give satisfactory results and thus EAs are capable of solving this class of difficult problems and the quality of results does not depend only on the problem being solved but also on the proper definition of the CF. In this contribution two different CFs were introduced and tested in the task of fast targeting and stabilization of desired periodic orbits. As can be seen from the optimization results presented here, they are extremely sensitive to the construction of used CF. Any small change in the design of $\mathrm{CF}$ can cause radical improvement of system behavior, but on the other hand can cause worsening of any other observed parameter, as in the case of $\mathrm{CF}_{\mathrm{TARG} 2}$ for $\mathrm{p}-2$ orbit.

There is no problem for the future research in defining much more complex $\mathrm{CF}$ comprising any criterion. Furthermore, parameter settings for EA were based on heuristic approach; therefore there is also possibility to bench them for the future research. According to all results shown here it is planned that the main activities will be focused on testing more complex cost functions together with searching for better settings of EA and certainly on testing of evolutionary deterministic chaos control in continuous-time and high-order systems.

\section{ACKNOWLEDGEMENT}

This work was supported by the grant NO. MSM 7088352101 of the Ministry of Education of the Czech republic and by grants of Grant Agency of Czech Republic GACR 102/06/1132 and GACR 102/05/0271.

\section{REFERENCES}

Just W., 1999, Principles of Time Delayed Feedback Control, In: Schuster H.G., Handbook of Chaos Control, WileyVch, ISBN 3-527-29436-8.

Kwon O.-J., 1999, Targeting and stabilizing chaotic trajectories in the standard map, Physics Letters A, 258, 229-236.

Ott E., C. Greboki, J.A. Yorke, 1990, Controlling Chaos, Phys. Rev. Lett. 64, 1196-1199.

Pyragas K., 1992, Continuous control of chaos by selfcontrolling feedback, Physics Letters A, 170, 421-428.

Pyragas K., 1995, Control of chaos via extended delay feedback, Physics Letters A, 206, 323-330.

Richter H. and K. J. Reinschke, 2000, Optimization of local control of chaos by an evolutionary algorithm, Physica $D, 144,309-334$.

Zelinka I., 2004, SOMA - Self Organizing Migrating Algorithm, In: New Optimization Techniques in Engineering, (B.V. Babu, G. Onwubolu (eds)), chapter 7, 33, Springer-Verlag, ISBN 3-540-20167X

Zelinka I., 2005a, Investigation on Evolutionary Deterministic Chaos Control - Extended Study, In proc. $20^{\text {th }}$ European Conference on modelling and simulation ECMS 2005, Riga, Latvia

Zelinka I., 2005b, Investigation on Evolutionary Deterministic Chaos Control, in proc. IFAC, Prague 2005

Senkerik R., Zelinka I., Navratil E., 2006a, Optimization of feedback control of chaos by evolutionary algorithms, in proc $1^{\text {st }}$ IFAC Conference on analysis and control of chaotic systems, Reims, France.

Senkerik R., Zelinka I., Navratil E., 2006b, Investigation on Evolutionary EDTAS Chaos Control, in proc. $20^{\text {th }}$ European Conference on modelling and simulation ECMS 2006, Bonn, Germany.

\section{AUTHORS BIOGRAPHIES}

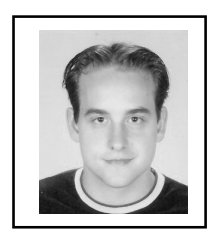

ROMAN SENKERIK was born in the Czech Republic, and went to the Tomas Bata University in Zlin, where he studied Technical Cybernetics and obtained his degree in 2004. He is now a Ph.D. student at the same university.

Email address: senkerik@fai.utb.cz

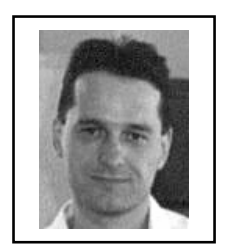

IVAN ZELINKA was born in the Czech Republic, and went to the Technical University of Brno, where he studied Technical Cybernetics and obtained his degree in 1995. He obtained Ph.D. degree in Technical Cybernetics in 2001 at Tomas Bata University in Zlin. Now he is an associated professor (Artificial Intelligence, Theory of Information) and head of department.

Email address: zelinka@fai.utb.cz .

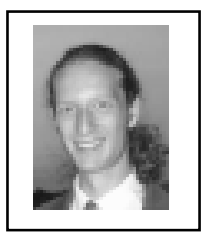

EDUARD NAVRATIL was born in the Czech Republic, reached his master degree in Mathematical Engineering at the Brno University of Technology in 2003. He is currently a Ph.D. student at Tomas Bata University in Zlin.

E-mail address: enavratil@fai.utb.cz 